\title{
Assistance in Learning and Teaching the English Article System: Diagnostics and Mnemonics
}

\author{
Douglas J. Wulf \\ Department of English, George Mason University, Fairfax, VA, USA \\ Email:dwulf@gmu.edu
}

Received 5 July 2016; accepted 12 August 2016; published 15 August 2016

Copyright (C) 2016 by author and Scientific Research Publishing Inc.

This work is licensed under the Creative Commons Attribution International License (CC BY). http://creativecommons.org/licenses/by/4.0/

(c) (i) Open Access

\begin{abstract}
Accurate use of the article system in English is a common problem even for advanced students of English as a second language (ESL). Articles are non-salient and there is much idiosyncrasy in their patterns of use. Extensive lists of rules and exceptions have been advanced to describe how to use articles, but such lists can be more confusing than helpful. A valuable pedagogical tool could be a simple set of diagnostics to check article patterns rapidly within their context of use coupled with mnemonics to help to summarize and recall various typical usage patterns. This paper advances such diagnostics and mnemonics drawing upon the etymology of the articles for inspiration. A small pilot study was also accomplished to indicate the potential pedagogical utility of this strategy for teaching and learning articles in English.
\end{abstract}

\section{Keywords}

English Grammar, English as a Second Language, Language Pedagogy, English Article System, Etymology, Diagnostics, Mnemonics

\section{Introduction}

The definite article the is the most common word in the English language; if we combine occurrences of $a$ and an, the indefinite article is the fourth most common (Sinclair, 1991). Additionally, we may consider the null article, indicating it visually with $\varnothing$. It is possible to have no overt article with non-count nouns or plural nouns (e.g., we need $\varnothing$ advice; $\varnothing$ rules are necessary). Master (1997) indicates that $\varnothing$ is more frequent than the, making $\varnothing$ English's most frequent free morpheme. This invisible morpheme expresses a measurement or quantity notion of some imprecise amount rather like some or a lot of (e.g., we need \{some/a lot of\} advice; \{some/a lot 
of\} rules are necessary). Grammar references acknowledge some/Ø only inconsistently. Whereas Hill \& Bradford (2000) label some a plural indefinite article, noting its frequent deletion, Stevenson (2005) lists $a$, an, and the as the only articles.

Although English language learners (ELLs) encounter articles continually, mastering them often remains elusive. Whitman (1974, p. 253) characterizes articles as "one of the most formidable problems to overcome in teaching English grammar to foreigners”. Covitt’s (1976) survey of ESL teachers ranked articles as the most difficult grammar point. Explicit instruction with corrective feedback appears ineffective in teaching articles (Kharma, 1981; Butler, 2002).

Articles are challenging for many reasons, yet much of the pedagogy for teaching articles is also flawed. As noted, an entire article pattern, use of the null article, is often downplayed or skipped. We can improve how relevant knowledge of articles is packaged for comprehension and retention and provide better practice and feedback.

Consider skill acquisition theory (SAT), which seeks to describe the acquisition of any skill, such as learning to play a sport, drive a car, or play a musical instrument. Under SAT, language acquisition has likewise been analyzed as an instance of skill learning (see, for example, DeKeyser, 1998). According to SAT, acquiring any skill is a multi-step process in the following way: 1) gain declarative knowledge of the skill (i.e., conscious understanding of the rules or patterns), 2) transfer this into procedural knowledge of skill performance (i.e., attempting to implement the rules or patterns), and 3) practice the skill extensively to build automaticity (i.e., smooth performance without conscious attention to the rules or patterns). Just as playing the piano can begin with much conscious and effortful attention but can ultimately result, after extended practice, in the pianist's hands seeming just to know what notes to play, so too can competency with a second-language grammatical pattern transition from halting application of the rules into smooth and automatic incorporation of the grammar in conversational use.

If this depiction of the acquisition of grammar as a skill is correct, the first step in acquiring article use successfully would be to understand the relevant rules and patterns clearly (i.e., the relevant declarative knowledge). However, ELLs may unfortunately never gain such firm declarative knowledge of articles to permit accurate procedural development. Rules and exceptions, such as the approximately seventy guidelines from Claire (1988), seem too elaborate to internalize and sometimes involve wider discourse context, not just sentence-level considerations. ELLs who are not in possession of accurate declarative knowledge would perhaps invent their own inaccurate interlanguage guidelines for article use, make wild guesses about articles, or frequently drop articles to avoid using a pattern whose purpose they do not understand. The result would then be proceduralization of a non-native-like pattern of article use (i.e., entrenched article errors in using English).

Again, if article grammar is acquired as a skill, as SAT indicates, then a major obstacle to success would be a failure to sufficiently and accurately convey rules and patterns of article use. We must reduce what is complicated to something simpler. I therefore propose a minimal set of diagnostics and mnemonics permitting faster evaluation of article use and better pattern retention. Since articles occur in non-salient unstressed syllables, ELLs performing group-work correction activities could help one another notice patterns, assuming they have been provided the requisite declarative knowledge. Then, in giving students feedback on their article use, instead of simply telling students that an article choice was incorrect or using such confusing terminology as definite versus indefinite, the mnemonics and diagnostics could be referenced in order to provide students with a comprehensible explanation of the error and the correction. I begin by summarizing the many details of article grammar and then encapsulate these in a diagnostic-mnemonic system. In a discussion of a small pilot study I conducted, this paper also reports on the results of a modest trial run in having ELLs use these diagnostics and mnemonics.

The diagnostics draw upon etymology for inspiration. Many grammar references mention the historical origins of the English articles. For example, Celce-Murcia and Larsen-Freeman (1999, p. 271) state, "The historical development of articles in English is similar to that of most other languages that have developed an article system: the definite article the derives from the demonstrative signaling distance (i.e., that), while the indefinite article derives from the numeral one". This etymology is typically regarded as a historical curiosity, but I emphasize its potential to provide, for diagnostic purposes, a temporary quasi-meaning to what are otherwise just functional elements in modern English, thereby revealing usage patterns. The etymology can provide at least something meaningful to what can seem otherwise meaningless and random.

Thus, the diagnostics portray how modern usage patterns first arose when these grammatical elements actually expressed content meaning. They allow the ELL to focus on the meaning of each utterance within the discourse 
context and, from this, make an appropriate article selection for that meaningful, contextualized, communicative situation. ELLs can avoid elaborate consideration of purely grammatical distinctions such as definite/indefinite, count/non-count, and the like.

In the following rather lengthy section, I cover the major points of article grammar. When described in a pointby-point way, each pattern and sub-pattern can be made understandable. However, when attempting to employ articles accurately, it seems a virtual impossibility to give conscious consideration to all the many factors involved in the system as a whole. This is why, after describing the system in a fully explicit way, I neatly encapsulate the same information in a short mnemonic poem with an accompanying name mnemonic.

Also, as a substitute for giving conscious consideration to the many factors at play in article selection, I provide three easy-to-use diagnostics. In the pilot study, ELLs were challenged to use the mnemonics and diagnostics to locate article errors in their own writing and in the writing of their peers and then make corrections. The study found that when there was documented evidence of the implementation of the diagnostics, there was also significant improvement in the location of article errors and their correction in contrast to attempts to locate and correct article errors made before being given the mnemonics and diagnostics as tools.

\section{Article Grammar}

\subsection{Common-Noun Markers}

We must now digress to provide an overview of article grammar to illustrate the declarative knowledge that we need the mnemonics and diagnostics to capture efficiently. The article system in English is rather complex with use of articles conditioned by a variety of considerations, some of which reach beyond the level of the phrase and the clause to consider the structure of the discourse as a whole.

Fundamentally, the function of an article is to modify a common noun, not a name (i.e., a proper noun). However, a word is not a common noun in isolation. Is fish a common noun? To answer, we must examine the overall context. In They fish, fish is a verb. In We need fish food, fish is a modifier in a compound. In "Fish" is my pet guppy, an atypical proper-noun interpretation is forced on this word. However, in I bought a fish, fish is (at last) a common noun, marked with the indefinite article $a$.

Indeed, one thing that articles do is to help indicate that a word is being used as a common noun. This is one reason why teaching vocabulary though decontextualized word lists has instructional shortcomings. Without the inclusion of function words, such as articles, and without examining language in its context of actual use, words in a list are really not being used as words in any meaningful way.

Note, for example, that fish only becomes a count noun (e.g., $\{$ A/Three $\}$ fish jumped) or a non-count noun (e.g., we put $\{\varnothing /$ some $\}$ fish in the stew) from context, provided in part through article use. The guidance Some nouns are either count or non-count is misleading. It fails to portray how articles shape interpretation. A salesperson may say, "You are getting a lot of car here!" (i.e., car as non-count noun). Lego is a company name, but it is possible to say "I bought some Legos" (a plural common noun referencing toy building blocks, capitalized only because of its derivation from the company's name).

Thus, we can in general only identify a word's grammatical identity from its usage context. We cannot properly categorize words in a list. English is quite flexible in this way. A word often has more than one unitary role, and articles help shape these roles. As Firth advised, "You shall know a word by the company it keeps" (1957/1968). This is why, for example, article diagnostics must be formulated in such a way as to inform an ELL which article to employ with a common noun, such as fish or car or Lego, not when the word is in a vocabulary list, but rather when the word is being used in a text or as part of a conversation.

\subsection{The Quantity Expressions $a / a n$ and $\varnothing$}

Describing the article system, as it is often done, as a contrast between a/an and the is pedagogically confusing. The indefinite article a/an indeed marks "indefiniteness", but so do other expressions, such as all numbers (e.g., three eggs — any three) and other quantity expressions (e.g., a lot of eggs—some indefinite quantity of them). The marks "definiteness", but so do possessives (e.g., my egg-the one I have) and demonstratives (e.g., that egg-the one over there).

Furthermore, we additionally have the null article $\varnothing$ in the article system. In fact, the contrast of a/an versus $\varnothing$ is much clearer to understand because both express quantity. Historically, $a$ and an were the word one (OED, 1989, [a, adj.]). Note that the letter $n$ is common to the words one and an, as these are etymologically derived 
from the same source. In Scotland, one is sometimes written ane (OED, 1989, [one adj. 14]). We can recover this association by stressing the article (e.g., I need a pencil-just one). Celce-Murcia and Larsen-Freeman (1999) note, “...it is in fact still possible to use one as a stressed emphatic variant of a/an: That's one big bug!” (p. 271). As we see, a/an is indefinite because, as noted, all numbers are indefinite.

Thus, a/an marks singular indefinite just like its etymological ancestor one: He has \{an/one\} egg. Ø is a blank created by dropping some, the plural counterpart of a/an: He has $\{\varnothing /$ some/a lot of $\}$ eggs. Like some, we use $\varnothing$ to indicate that a noun is non-count: He has $\{\varnothing /$ some/a lot of $\}$ egg on his face. Thus, a/an is for counting (to one) and $\varnothing$ (or its overt realization some, a lot of, etc.) is also for counting (a vague quantity) or measuring (a vague amount).

As with a lot of, we have other quantity/measurement expressions containing an article, often the indefinite (e.g., a pound of), but also definite and null (e.g., the majority of; $\varnothing$ lots of). Unfortunately, some has another meaning that is approximately the meaning of the indefinite article (e.g., \{Some/A\} man arrived-I don't know his name.), whereas a lot of avoids this ambiguity. Therefore, although we can conceptualize the null article $\varnothing$ as a deleted occurrence of some, we can also associate it (and approximately paraphrase its meaning) with some different vague expression of quantity, such as a lot of.

\subsection{The Demonstrative the}

The definite article the is quite distinct from $a / a n$ and $\varnothing$. Its function is not the counting or measuring function of a quantity expression, but rather the pointing function of a demonstrative expression. Note that $a / a n$ and $\varnothing$ occur syntactically where quantities occur (e.g., \{a/one/three/many/Ø\} fish), but the occurs in advance of this position: the \{one/three/many\} fish. We do not say *the a fish simply because one appears instead: the one fish.

Etymology can again prove helpful. In the history of English, the and that were two forms of the same demonstrative (OED, 1989, [the, adj., pron.2, and n.2]). At a time when English had grammatical gender (like modern German), the marked masculine and that marked neuter. Note the common occurrence of th and the similarity of meaning between Park the car and Park that car. We can thus approximately regard the as a fifth demonstrative alongside this, that, these and those.

In particular, the is used to point something out by introducing a description sufficiently clear for the context. That is, the is used to generate a definite description. For example, if we have a dozen eggs, a person may say, "Take the egg closest to you”, and then say, "Crack the egg”. In the first statement, it is necessary to describe in more detail which of the twelve eggs to take. However, by the second statement, it has become clear that "the egg” references that particular egg taken, even though eleven other eggs are still present. The definite description can, at this point, be quite brief: “the egg”.

A common noun marked with the points out some thing (or group of things) by generating a definite description, a construction that functions much like a name. In The teacher of my English class is Adele Camus, we refer to an individual by name (Adele Camus) as well as by definite description (The teacher of my English class). Indeed, definite descriptions are so like names that some definite descriptions have become conventionalized as "names". Thus, we may identify a country by name (e.g., France), by definite description (e.g., the United States of America), or sometimes by either (e.g., Saudi Arabia; the Kingdom of Saudi Arabia).

\subsection{Tokens and Types}

One confusing complication in the article system is that common nouns can reference either tokens (e.g., $I$ saw two tigers at the zoo: Sam and Molly) or types (e.g., I saw two tigers at the zoo: the Bengal and the Sumatran). We use all article options not only to reference tokens, but also to reference types: an egg is round (any one of that type), Ø eggs are round (a lot of, maybe all of that type) or, in formal usage, the egg is nutritious (that type of thing).

This distinction is conspicuously apparent when typically non-count nouns are pluralized, expressing types: We examined two sugars: fructose and glucose (two types). As a pourable mass quantity, sugar is not tokenized, but when referencing types of sugar, these types can be counted. Also, sugar can be counted if referencing portions: I put two sugars (two packets) in my coffee.

The token/type distinction also arises in another way. We may tell a sick person, "Go to the doctor!" without having a particular doctor (a token) in mind. We reference only that type of professional. We may announce going on an aimless shopping trip with I'm going to the store (i.e., that type of destination, perhaps without 
having a particular store in mind). Thus, it is possible for the type being referenced to be definite, thereby justifying use of the, without there being any definite token involved.

\subsection{Bridging}

A rule-of-thumb often given to ELLs is to use a/an when mentioning something for the first time, but the thereafter (e.g., I saw a house. I walked into the house and entered the kitchen). Here, the first mention of house takes $a$, but the next takes the. However, this does not explain how kitchen takes the with its first mention. Clark (1977) calls this phenomenon bridging.

Note that it would be ponderous first to indicate that the house previously mentioned had a kitchen and it was into the kitchen that was just mentioned that I walked. Instead, once the house is established in the discourse, we are able to "bridge" our understanding of the kitchen as that kitchen of the aforementioned house.

When and how English speakers employ bridging is subtle, but being aware that bridging occurs is a valuable step to mastering this discourse pattern. Like the issue of the token/type distinction, the bridging phenomenon can be explained to ELLs, but it is then a challenge to determine how ELLs may gain a sense of when and how native speakers use such patterns conversationally. It is my hope that the mnemonic/diagnostic system outlined in this paper may serve as a basic framework for building procedural knowledge of such subtle usage issues.

\subsection{Verbal Nouns}

Infinitive and gerundive phrases can play the same role as a typical noun phrase, such as serving as the subject of a clause, as in $\{$ To cross the river/Crossing the river $\}$ is difficult. Infinitives do not employ articles, and gerundives only rarely employ the in formal style (e.g., The crossing of the river is difficult). Infinitives and gerundives do not follow the typical structure for noun phrases, yet they are routinely employed in language. In addition, since an infinitive phrase or gerundive phrase contains a verb, this verb can itself take an object, and this object noun phrase can take an article, as we see with the river in To cross the river and Crossing the river.

\subsection{Special Prepositional Phrases}

Common nouns in a somewhat limited set of prepositional-phrase expressions take no article, as with for example (*for an example). Others are at school, on foot, by mistake, and in question. These nouns can take articles within similar prepositional phrases (e.g., at a school), but the meaning changes (de Swart \& Zwarts, 2009). Being aware that this pattern exists is certainly the first step to acquiring it, though proceduralization of this pattern can only come with practice and accurate feedback.

\subsection{Indefinite Article Variants}

The indefinite article is $a$ before a consonant sound (e.g., $a$ bicycle) and an before a vowel sound (e.g., an art).

\section{Mnemonics and Diagnostics to Provide Declarative Knowledge of Articles}

The goal is now to compress these many elaborate details of article grammar into a streamlined diagnostic-mnemonic system. The diagnostics must be simple enough to employ when using common nouns in context. The mnemonics must be brief, yet capable of portraying large portions of article use in English overall.

\subsection{A Name Mnemonic}

Names are not difficult to understand, provided the distinction between true names and definite descriptions (sometimes used as conventionalized names) is clear. Yet, the range of name usage can be reinforced through a mnemonic within which each ELL may select personally relevant examples. Figure 1 provides one version. This covers various kinds of names, but is not exhaustive. Note that some names are not capitalized.

Suppose an ELL writes *I study the graphic design. This error can be compared with the ELL's mnemonic, thereby indicating the nature of the mistake: graphic design names a field of study (like math). That is, the ELL must state I study graphic design for the same reason that $I$ study math is in the mnemonic, which is that names do not take articles.

A notable example of this particular article error is how Ivana Trump referred to her then husband Donald 


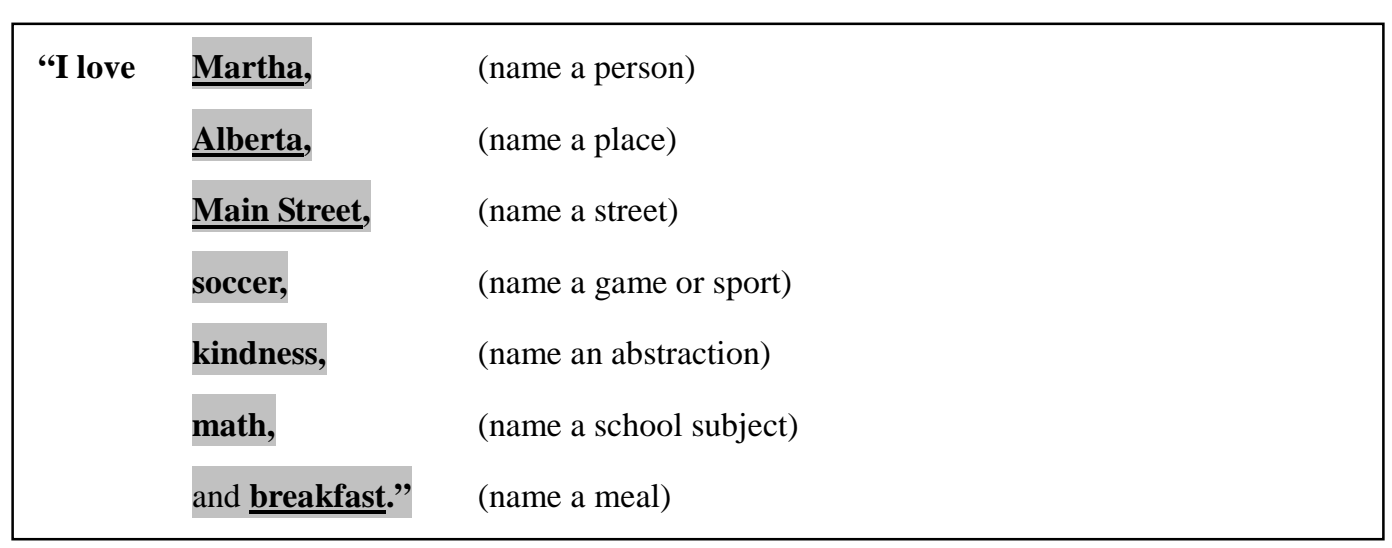

Figure 1. A name mnemonic.

Trump as “The Donald”. As Ivana Trump explains, "When I came to live in New York, I really had to learn the language from the beginning almost. Some things come easily, some things don't. And for whatever reason, probably because I was going at my usual turbo speed, I started putting 'The' in front of most people's names. Yes, you know the outcome- 'The Donald' just slipped off the tongue, and now it seems to be making its ways to the political history books" (quote from Argetsinger, A. "Why Does Dveryone Dall Donald Trump 'The Donald?' It's an Interesting Story” from The Washington Post, September 1, 2015, viewed at https://www.washingtonpost.com/news/arts-and-entertainment/wp/2015/09/01/why-does-everyone-call-donald-t rump-the-donald-its-an-interesting-story/).

\subsection{Article Diagnostics}

For rapidly testing the contextual applicability of articles, I propose the diagnostics in Figure 2. Although not foolproof, etymology suggests that more effective diagnostics cannot be devised. When employed, an ELL can sweep aside the need to give conscious consideration to distinctions of definite/indefinite, singular/plural, count/noncount, token/type, bridging, and others. The ELL simply checks which diagnostic of the three diagnostics works best in terms of the contextualized meaning with the common noun in question in the context in which the noun is found. The diagnostic does not need to coincide perfectly with the meaning, but only needs to fit the intended meaning better than the other two diagnostic alternatives. The diagnostics temporarily convert these purely functional elements of English into meaningful content expressions. Through continued use, the diagnostics also gradually serve to illuminate the rationale of the article system of English as a whole.

Many languages have no articles, and those that do may use them in ways different from English. However, expressions for one, that, and some/a lot of are all near universals in the world's languages (Horrocks \& Stavrou, 1987). Thus, virtually all ELLs should have intuitions about native-language counterparts of these expressions, making them a foundation upon which to conceptualize and swiftly diagnose English article use. Again, employing some as the null article diagnostic must be done with care, as it is must be understood as the interpretation of some as an indefinite quantity expression, similar to a lot of (e.g., I need some eggs and some sugar), and not the interpretation of some involving non-specific reference, similar to a/an (e.g., I see some man over there; I see a man over there). This is why a lot of is included as an alternative diagnostic for the null article.

\begin{tabular}{cll|}
\hline Article & & Diagnostic \\
\hline $\mathbf{a} / \mathbf{a n}$ & the indefinite article & Try “one” \\
$\boldsymbol{\emptyset}$ & the null article & Try “some” "or “a lot of" \\
the & the definite article & Try “that" or "those” \\
\hline & Additional note: The definite article "the" introduces a definite description. \\
\hline
\end{tabular}

Figure 2. Article diagnostics. 
To illustrate use of the diagnostics, consider the clause He found fish, in which the article has not yet been specified. The article selected would depend on the context in which the clause is being used. If the contextualized meaning is most closely paraphrased by He found one fish, then the clause should be He found a fish (e.g., He found a fish to put in his fish tank or He found a fish that was an unknown species). However, if the contextualized meaning is most closely paraphrased by He found (some/a lot of) fish, then the clause should be He found $\varnothing$ fish (e.g., He found Ø fish to put in the stew; He found Ø fish to put in his fish tank; He found Ø fish that were unknown varieties). Lastly, if the contextualized meaning is most closely paraphrased by He found that fish or He found those fish, then the clause should be He found the fish (e.g., He found the fish that he most wanted for his fish tank or He found the fish that would be best in the stew or He found the fish that is the most endangered species in the region). By rapidly giving consideration to these diagnostic paraphrases, meaning can be temporarily introduced for what would otherwise be purely functional elements, thereby allowing an ELL to make sense of the choice of article.

\subsection{An Article Mnemonic}

Figure 3 encapsulates much of the article system of English in a short mnemonic: a seven-line, 36-word poem (including the title) that I authored.

Mnemonics often employ poetry (e.g., Thirty days hath September, April, June, and November). Also, although articles occur in unstressed syllables, in order to retain the meter, ELLs cannot drop articles as they might if the mnemonic were prose. Here follows a line-by-line explanation.

L0 The Bicycle Poem provides the poem's title, which is not a true name, but rather a definite description, introduced with The. Bicycle (capitalized for the title) serves as a modifier expression. This again emphasizes that we cannot categorize a word from a vocabulary list, such as bicycle, as a common noun automatically. The role that a word plays in language ultimately depends on its use in context.

L1 I bought a bicycle introduces a new discourse element with a/an (i.e., Recall the diagnostic I bought one bicycle).

L2 I named it "Bicycle" forces an unusual proper-noun interpretation via the use of no article at all. The name "Bicycle" is capitalized and takes no article (i.e., Recall the name mnemonic I love Martha. The counterpart clause would be I love Bicycle, assuming the name of this bicycle is "Bicycle”).

L3 I took the bicycle apart completes the discourse pattern begun in L1 with the second mention of a discourse element, now taking the (i.e., Recall the diagnostic I took that bicycle apart-the one previously mentioned). This definite description is short (the bicycle) since we need not distinguish it from any other bicycles at this point in the discourse. This definite description works much like the name "Bicycle".

L4 There's bicycle now all over the floor forces a grammatical, though atypical, non-count interpretation (i.e., Recall the diagnostic There's a lot of bicycle now all over the floor). Many such expectation-reversing examples exist. Consider, for instance, $\varnothing$ chewed-up pencil (Master, 2002) and Give me some pillow (Michaelis, 2003). In one adventure of Winnie-the-Pooh, Pooh gets a honey jar stuck on his head, but using jar with the null article $\varnothing$, A. A. Milne writes that Pooh “...tried to climb out of the Trap, but as he could see nothing but jar, and not much of that, he couldn’t find his way” (from A. A. Milne, Winnie-the-Pooh, 1926, published by E. P. Dutton). A commonly encountered pattern is how meat can be referenced: They added $\varnothing$ chicken to the soup. By including this pattern in the mnemonic poem, ELLs can come to realize the consequences of dropping articles since blanks (i.e., the null article $\varnothing$ with common nouns or the avoidance of all articles with names) also play grammatical roles in English.

\begin{tabular}{|ll|}
\hline L0 & The Bicycle Poem \\
L1 & I bought a bicycle \\
L2 & I named it "Bicycle" \\
L3 & I took the bicycle apart \\
L4 & There's bicycle now all over the floor \\
L5 & By bicycle now I'll travel no more \\
L6 & Yes, fixing bicycles is an art \\
\hline
\end{tabular}

Figure 3. An article mnemonic. 
In L4, we also encounter the floor, which contrasts with a bicycle/the bicycle from L1/L3. Via bridging, without any prior use of the indefinite article with this noun (i.e., a floor), the floor can be understood to reference that floor where the bicycle was disassembled. It would be ponderous first to use the indefinite article (a quantity expression approximately indicating one) to indicate that there was one floor there and only then use the definite article (a demonstrative expression approximately indicating that) to indicate that it was that particular floor involved. Bridging is thus a phenomenon of efficient language use.

L5 By bicycle now I'll travel no more provides a bare noun in a prepositional phrase, thereby acknowledging that this pattern exists in the grammar. By bicycle describes a kind of travel (i.e., bicycle travel), expressing a notion similar to I'll bicycle no more. If an ELL ever wonders why English speakers can say for instance instead of *for an instance, the expression by bicycle can be noted as an example of this grammatical pattern.

L6 Yes, fixing bicycles is an art has a verbal noun phrase (a gerundive phrase), and these typically do not employ articles, though in formal style, use of the is possible (i.e., The fixing of bicycles). This verbal noun phrase serves as the subject of this clause. Another verbal noun variant is an infinitive, but infinitives do not take articles.

Also, in L6, within the gerundive phrase itself, we encounter $\varnothing$ bicycles, an example of type reference (i.e., fixing bicycles, any quantity of that kind of thing, is an art) rather than token reference.

Lastly, in L6, we encounter an art, which, together with $a$ bicycle from L1, illustrates the distinction of $a$ versus an. Thus, the mnemonic poem also manages to demonstrate this phenomenon of allomorphic variation in the indefinite article’s forms.

Once ELLs are aware of these patterns and grasp them in a general way, this mnemonic poem can serve as a reminder of them as well as to reference them later in an efficient fashion. If an ELL writes *I study English in the school, the issue can be associated with by bicycle from this poem, making the correction I study English in school comprehensible. The mnemonic thus provides an organizing framework for categorizing and discussing complex article patterns.

\section{Practice in Building Procedural Knowledge of Articles}

Obviously, ELLs cannot master article use simply by being provided these mnemonics and diagnostics. These are merely tools to package the prerequisite declarative knowledge of article grammar in a manageable format. The next phase in the acquisition process is development of procedural knowledge, which eventually leads to building automaticity of use. For this, practice and feedback is required, and group work can be a part of this as peers can help one another notice the non-salient patterns of article use.

\subsection{A Pilot Study: Description of the Study}

As an initial investigation into the utility of using these diagnostics and mnemonics with article practice, I conducted a small pilot study with 10 participants, all ELLs in an ESL program. My aim was simply to locate any evidence that use of the diagnostics and mnemonics as tools could have a positive impact on article acquisition.

The ages of the participants ranged from 18 to 28 years, with five male and five female participants. Eight were native speakers of Arabic and two of Korean. Groups of 2 - 3 members were formed based on English proficiency. This was accomplished by forming each group with classmates from the same proficiency-level class. At the start of the semester, participants had been placed in their classes based on their performance on a proficiency test. In this way, there were two intermediate groups, one high-intermediate group, and one advanced group. I had never met any of the participants previously.

In the pre-test phase, each participant was given one of his/her own uncorrected class essays and a class essay written by each member of that participant's group. All essays had been written earlier that same semester. I had transcribed each essay uncorrected into a word-processed document to control for differing handwriting readability. With no external guidance or direction, each group was simply tasked to attempt to correct article errors in their group's essays. Corrections could be made by inserting an article, deleting an article, or changing one article to another. Each group worked in isolation.

After the pre-test, I provided diagnostic/mnemonic instruction. Since the study's aim was to streamline article teaching, instruction was strictly limited to one hour only. I used realia, such as plastic eggs and packets of two types of sugar to illustrate such notions as count/non-count, token/type, and other concepts as described in Section 2 of this paper. The grammar was then associated with the mnemonics and the diagnostics, as described in 
Section 3 of this paper.

For the post-test, the pre-test procedure was repeated, but with different retyped, uncorrected class essays, written by the same participants earlier in the semester. In order to keep the pre-test and post-test tasks comparable, it was important that groups had the same number of errors to attempt to correct in the pre-test as they did in the post-test. By simply removing a portion of text from the end of either the participants' pre-test or post-test essays, as needed, the pre-test and the post-test for each group was made comparable in the number of article errors to be located and corrected. Again, each group worked in isolation. However, participants were now asked to apply the diagnostics in making their corrections.

Yet, it was not enough simply to request that the participants employ the diagnostics in making corrections. Rather, it was crucial that some visible record be generated in order to verify that the diagnostics were indeed employed. Therefore, participants were also taught to document the correction process in a specific way. Figure 4 shows the steps requested.

Figure 5 is a sample text showing how corrections might be marked with underlines and check marks. Underlines and checkmarks being added correctly to the text would be visible evidence of the correction procedure being followed successfully, whereas a lack of underlines and checkmarks or incorrect use of underlines and checkmarks would be evidence of a failure to follow the correction procedure.

Before the post-test, I helped participants practice documenting corrections in this way using additional examples of class essays that the participants had written that semester. Expressions in parentheses in Figure 5 would not be written in their corrected essays, but only show how ELLs might paraphrase or diagnose common nouns as they worked through the text. That is, ELLs were only required to add underlines, checkmarks, and article corrections, as needed.

\begin{tabular}{|c|c|}
\hline Step 1 & Underline each common noun. Do not underline names. Instead, check off names. \\
\hline & $\begin{array}{l}\text { NOTE: For compounds, underline the entire compound (e.g., car wash, not just car } \\
\text { or wash).Do not underline gerunds or infinitives (e.g., for Saving moneyis difficult, } \\
\text { underline } \underline{\text { money, not saving). }}\end{array}$ \\
\hline \multirow[t]{2}{*}{ Step 2} & Check off common nouns already marked with a demonstrative or a possessive. \\
\hline & $\begin{array}{l}\text { These already point things out so do the work of the. Also, a noun that has a quantity } \\
\text { or measurement expression excludes use of a/an and } \varnothing \text {, but not the. This is because } \\
\text { a/an and } \varnothing \text { themselves express quantity or measurement. }\end{array}$ \\
\hline Step 3 & Apply the article diagnostics and correct as necessary. \\
\hline
\end{tabular}

Figure 4. Documentation of the correction process.

\begin{abstract}
One major problem $\sqrt{ }$ (quantity expression - one) with using an express car wash (one express car wash, one of those) to clean a car (one car, one of those) is that it may damage your antenna $\sqrt{ }$ (possessive - your). Antennas (Some antennas, Some of them) are sometimes damaged when you use a car wash service (one car wash service, one of those) because they can be bent by the brushes (those brushes, those ones) used to spread soap (some soap, some of that). That problem $\sqrt{ }$ (demonstrative - That) happened to my wife $\sqrt{ }$ (possessive - my)'s friend $\sqrt{ }$ (possessive - my wife's), Pamela $\sqrt{ }$ (name) so, be on guard (special prepositional phrase use) against that.
\end{abstract}

Figure 5. An example text.

\title{
4.2. The Pilot Study: Results
}

Table 1 provides pre-test results. Since higher-proficiency ELLs wrote longer texts, their texts contained many 
more sentences with articles (and, thus, many more common nouns requiring articles), and therefore their texts also contained more article errors.

Despite taking as much time as desired, no group corrected more than two errors net. That is, the net correction was the correction of preexisting errors minus any overcorrection of originally correct forms to something incorrect. Percentages for lower proficiency groups were higher merely because the net correction of a small number of errors represented a larger percentage change to that error count. Pre-test results suggest that unguided attempts to correct article errors are not worthwhile at any proficiency level.

It should be noted that in their acquisition of English, these ELL have certainly had vast exposure to articles, as these are ubiquitous grammatical elements in English. In addition, these ELLs have certainly all received formal instruction on the grammar of articles. However, all of these ELLs appeared to lack sufficiently in-depth declarative knowledge of subtle article usage patterns to implement corrections procedurally in their own writing or in their classmates' writing to any significant extent.

After being taught the diagnostic/mnemonic system for less than one hour, they attempted the post-test correction, and Table 2 depicts their performance in documenting their use of diagnostics and mnemonics through underlining and check marking of common nouns in the texts.

The second line indicates the total number of underlines and check marks expected if the documenting procedure were followed accurately. The last two lines indicate the net percentage of correct underlining and check marking, respectively. Negative percentages indicate that erroneous check marking exceeded correct check marking.

Intermediate Group 2 did well at the underlining task, but Intermediate Group 1 was less successful. Neither intermediate group was successful with check marking, with Intermediate Group 1 introducing many extraneous checks. This is evidence that neither intermediate group was able to analyze their texts sufficiently in order to employ the diagnostic tests successfully.

The advanced group began employing underlining and check marking in their post-test, but then stopped. Some erroneous checks gave this group also a negative score for that task. The advanced students seemed to have understood the diagnostics and mnemonics, but unfortunately did not implement the system as well as they probably could have done. Apologizing that they had run out of time to devote to the study, the advanced group hurried through the post-test phase and left. Nonetheless, they did manage to implement some corrections to their post-test essays.

Of the four groups, only the high-intermediate group documented both underlining and check marking fairly successfully. Although far from high accuracy in analyzing the common nouns in their texts, there was at least some evidence of understanding the procedures and being able to follow them.

Finally, Table 3 provides results of post-test article corrections.

Post-test results for the intermediate groups were consistent with pre-test results, although the advanced group indeed showed some improvement. I interpreted this to mean that the two intermediate groups did not understand

Table 1. The pre-test (the control trial) error corrections.

\begin{tabular}{|c|c|c|c|c|}
\hline Group & Intermediate 1 & Intermediate 2 & High Intermediate & Advanced \\
\hline Number of Students & 2 & 2 & 3 & 3 \\
\hline Number of Errors & 20 & 19 & 26 & 61 \\
\hline Net Correction & +2 & +2 & +1 & +1 \\
\hline Percent Changed & $+10 \%$ & $+11 \%$ & $+3.8 \%$ & $+1.6 \%$ \\
\hline
\end{tabular}

Table 2. The post-test (the experimental trial) evidence of using the diagnostic system.

\begin{tabular}{|c|c|c|c|c|}
\hline Group & Intermediate 1 & Intermediate 2 & High Intermediate & Advanced \\
\hline Number of Students & 2 & 2 & 3 & 3 \\
\hline Underlines/Checks & $84 / 10$ & $117 / 25$ & $80 / 25$ & $195 / 80$ \\
\hline Net Percent Underlined & $23 \%$ & $81 \%$ & $60 \%$ & $9 \%$ \\
\hline Net Percent Checked & $-200 \%$ & $0 \%$ & $20 \%$ & $-5 \%$ \\
\hline
\end{tabular}


Table 3. The post-test (the experimental trial) error corrections.

\begin{tabular}{ccccc}
\hline Group & Intermediate 1 & Intermediate 2 & High Intermediate & Advanced \\
\hline Number of Students & 2 & 2 & 3 & 3 \\
Number of Errors & 20 & 19 & 26 & 61 \\
Net Correction & +1 & +2 & +12 & +5 \\
Percent Changed & $+5 \%$ & $+11 \%$ & $+46 \%$ & $+8.2 \%$ \\
\hline
\end{tabular}

how to use the system, whereas the advanced group showed clear improvement in their ability to correct article errors, despite not documenting use of the system as they were tasked to do. Although this group was only able to give a marginal effort in their post-test, their performance nonetheless improved from their pre-test phase.

However, the most dramatic improvement was with the high-intermediate group, which was also the only group to document somewhat successfully that they had attempted to follow the correction procedures rigorously. As indicated in Table 3, this group located and successfully corrected nearly half the article errors in its set of texts. Though only a small pilot study, from this positive result, we can at least see some indication of the potential utility of such diagnostics and mnemonics for usefully formulating the declarative knowledge relevant to eventually proceduralizing use of the article system in English.

\section{Conclusion}

The modest improvement of the advanced group and the substantial improvement of the high-intermediate group in this study suggest that these diagnostics could be helpful in fostering a declarative-to-procedural knowledge approach in the acquisition of the article system of English. This could be effective even though the language elements in question are notoriously fraught with exceptions and ambiguities.

Obviously, this pilot study was only designed to see if there might be any immediate effect after just an initial exposure to this diagnostic-mnemonic system. The participants had only just been introduced to this system and this was their very first attempt at applying it. Yet, given that a positive result was observed in two of the four groups, I now look forward to conducting a longitudinal study to investigate potential positive benefits over a longer course of time.

Diagnostic-mnemonic systems may also prove useful for improving other points of grammar. They may serve as frameworks for teachers and students to discuss errors and usage patterns. Such systems allow ELLs to focus their conscious attention not on abstract grammatical distinctions, but rather on the meaning of an utterance within the immediate discourse-level context.

\section{Acknowledgements}

The author thanks Adele Camus, Ellen Kohn, Justin Voigt, and all the instructors and administrators at George Mason University’s INTO Academic English.

\section{References}

Butler, Y. (2002). Second Language Learners’ Theories on the Use of English Articles: An Analysis of the Metalinguistic Knowledge Used by Japanese Students in Acquiring the English Article System. Studies in Second Language Acquisition, 24, 451-480. http://dx.doi.org/10.1017/s0272263102003042

Celce-Murcia, M., \& Larsen-Freeman, D. (1999). The Grammar Book: An ESL/EFL Teacher's Course (2nd ed.). Boston, MA: Heinle and Heinle.

Claire, E. (1988).Three Little Words: A, An, and The: A Foreign Student's Guide to English Articles. McHenry, IL: Delta Publishing Co.

Clark, H. H. (1977). Bridging. In P. N. Johnson-Laird, \& P. C. Wason (Eds.), Thinking: Readings in Cognitive Science (pp. 411-420). Cambridge: Cambridge University Press.

Covitt, R. I. (1976). Some Problematic Grammar Areas for ESL Teachers. Master's Thesis, Los Angeles: UCLA.

de Swart, H., \& Zwarts, J. (2009). Less Form-More Meaning: Why Bare Singular Nouns Are Special. Lingua, 119, 280295. 
http://dx.doi.org/10.1016/j.lingua.2007.10.015

DeKeyser, R. M. (1998). Beyond Focus on Form: Cognitive Perspectives on Learning and Practicing a Second Language. In C. Doughty, \& J. Williams (Eds.), Focus on Form in Classroom Second Language Acquisition (pp. 42-63). New York, NY: Cambridge University Press.

Firth, J. (1957). A Synopsis of Linguistic Theory, 1930-55. In Studies in Linguistic Analysis (pp. 1-31). Special Volume of the Philological Society. Oxford: Blackwell. [Reprinted as Firth (1968)]

Firth, J. (1968). A Synopsis of Linguistic Theory, 1930-55. In F. R. Palmer (Ed.), Selected Papers of J. R. Firth (1952-59) (pp. 168-205). London: Longmans.

Hill, S., \& Bradford, W. (2000). Bilingual Grammar of English-Spanish Syntax. Lanham, MD: University Press of America.

Horrocks, G., \& Stavrou, M. (1987). Bounding Theory and Greek Syntax: Evidence for wh-Movement in NP. Journal of Linguistics, 23, 79-108. http://dx.doi.org/10.1017/S002222670001104X

Kharma, N. (1981). Analysis of the Errors Committed by Arab University Students in the Use of the English Definite/Indefinite Articles. IRAL, 19, 333-345. http://dx.doi.org/10.1515/iral.1981.19.1-4.333

Master, P. (1997). The English Article System: Acquisition, Function, and Pedagogy. System, 25, 215-232. http://dx.doi.org/10.1016/S0346-251X(97)00010-9

Master, P. (2002). Information Structure and English Article Pedagogy. System, 30, 331-348. http://dx.doi.org/10.1016/S0346-251X(02)00018-0

Michaelis, L. (2003). Word Meaning, Sentence Meaning, and Syntactic Meaning. In H. Cuyckens, R. Dirven, \& J. R. Taylor (Eds.), Cognitive Approaches to Lexical Semantics (pp. 93-122). Berlin/New York, NY: Mouton de Gruyter. http://dx.doi.org/10.1515/9783110219074.163

Oxford English Dictionary (OED). 1989. New York, NY: Oxford University Press.

Sinclair, J. (Ed.) (1991). Corpus, Concordance, Collocation. Oxford, UK: Oxford University Press.

Stevenson, J. (2005). The Pocket Idiot's Guide to Grammar and Punctuation: A Handy Reference to Resolve All Your Grammatical Problems. New York, NY: Penguin.

Whitman, R. (1974). Teaching the Article in English. TESOL Quarterly, 8, 253-262. http://dx.doi.org/10.2307/3586169

\section{Submit or recommend next manuscript to SCIRP and we will provide best service for you:}

Accepting pre-submission inquiries through Email, Facebook, LinkedIn, Twitter, etc.

A wide selection of journals (inclusive of 9 subjects, more than 200 journals)

Providing 24-hour high-quality service

User-friendly online submission system

Fair and swift peer-review system

Efficient typesetting and proofreading procedure

Display of the result of downloads and visits, as well as the number of cited articles

Maximum dissemination of your research work

Submit your manuscript at: http://papersubmission.scirp.org/ 